\title{
Substrate reduction therapy in three patients with Gaucher disease
}

\author{
Soo Hyun Kim¹, Eungu Kang', Yoon-Myung Kim¹, Gu-Hwan Kim², In-Hee Choi², Jin-Ho Choi', \\ Han-Wook Yoo ${ }^{1,2}$, and Beom Hee Lee ${ }^{1,2, *}$ \\ ${ }^{1}$ Department of Pediatrics, Asan Medical Center Children's Hospital, University of Ulsan College of Medicine, Seoul, Korea \\ ${ }^{2}$ Medical Genetics Center, Asan Medical Center Children's Hospital, University of Ulsan College of Medicine, Seoul, Korea
}

\begin{abstract}
Purpose: Gaucher disease (GD) is the most common lysosomal storage disease caused by beta-glucocerebrosidase (GBA) deficiency. Oral substrate reduction therapy with miglustat $\left(\right.$ Zavesca $^{\circledR}$ ) was approved for the treatment of adults with GD type 1, for whom enzyme replacement therapy (ERT) is unsuitable or not a therapeutic option. In this study, we report the effect of miglustat $\left(\right.$ Zavesca $\left.^{\circledR}\right)$ in three Korean GD patients.

Materials and Methods: Clinical findings comprising age at diagnosis, presenting signs, laboratory findings at diagnosis, GBA activity and mutations, and clinical courses of the three patients were reviewed.

Results: Miglustat was administered to three patients who reported allergic reactions during intravenous imiglucerase infusions. One patient withdrew after 15 months of miglustat administration owing to continuous elevation of disease biomarker levels (chitotriosidase, acid phosphatase, and angiotensin-converting enzyme). Poor adherence to medication was suspected but was denied by the patient. In the other two patients, platelet count and levels of hemoglobin and other biomarkers remained stable during miglustat administration. However, they suffered from severe diarrhea and weight loss, which led to miglustat discontinuation after 1 and 12 months of administration.

Conclusion: Our study shows that although miglustat is suggested to GD patients as an alternative treatment to ERT, significant adverse reactions may lead to discontinuation of miglustat. In addition, it is difficult to monitor the drug adherence.
\end{abstract}

Key words: Gaucher disease, Miglustat.

\section{Introduction}

Gaucher disease (GD) is one of the most common autosomal recessive lysosomal storage diseases caused by beta-glucocerebrosidase (GBA) deficiency. Accumulation of glucocerebroside, mainly within tissue macrophages, results in diverse clinical manifestations of the disease, including hepatosplenomegaly, anemia, thrombocytopenia, and skeletal and neuronopathic symptoms [1].

Usually, GD is suspected during clinical examination by the incidence of unexpected anemia, thrombocytopenia, and organomegaly. A bone marrow or liver biopsy can assist the diagnosis by identifying typical Gaucher cells, namely, macrophages filled with lipid materials. The diagnosis is confirmed by detection of low enzymatic activity of GBA in peripheral blood and/or identification of recessive mutations

Received: 22 June 2016, Revised: 8 September 2016, Accepted: 16 September 2016, Published: 31 December 2016

*Corresponding author: Beom Hee Lee, M.D., Ph.D.

Department of Pediatrics, Asan Medical Center Children's Hospital, University of Ulsan College of Medicine, 88 Olympic-ro 43-gil, Songpa-gu, Seoul 05505, Korea.

Tel: +82-2-3010-5950, Fax: +82-2-473-3725, E-mail: bhlee@amc.seoul.kr

Conflict of interest: The authors declare that they do not have any conflicts of interest.

(c) This is an open-access article distributed under the terms of the Creative Commons Attribution Non-Commercial License (http://creativecommons.org/licenses/by-nc/4.0/) which permits unrestricted non-commercial use, distribution, and reproduction in any medium, provided the original work is properly cited.

(c) Copyright 2016 by the Korean Society of Medical Genetics www.e-kjgm.org 
in the GBA gene [2]. Furthermore, biomarkers such as acid phosphatase, angiotensin-converting enzyme (ACE), and chitotriosidase represent disease activity and can be useful indicators of GD progression [3].

There are three subtypes of GD, characterized by the presence of neurologic symptoms, age at identification, and disease progression rate: non-neuronopathic GD (type 1), acute neuronopathic GD (type 2), and chronic neuronopathic GD (type 3) [4].

After the introduction of recombinant GBA in 1994, enzyme replacement therapy (ERT) has significantly improved the clinical outcome of GD. To date, four recombinant enzymes have been introduced, namely, imiglucerase (Cerezyme ${ }^{\circledR}$, Genzyme Corp., Cambridge, MA, USA [5]; Abcertin ${ }^{\circledR}$, ISU Abxis, Seongnam, Korea [6]), velaglucerase alfa (VPRIV ${ }^{\circledR}$, Shire Human Genetic Therapies, Lexington, MA, USA) [7], and taliglucerase alfa (Elyso ${ }^{\circledR}$, Pfizer, New York, NY, USA) [8]. A previous study has shown that ERT alleviates GD-associated anemia, thrombocytopenia, organomegaly, bone pain, and bone crises and delays disease progression [9]. However, because recombinant enzymes cannot cross the blood-brain barrier, they cannot prevent neurological deterioration in patients with neuronopathic GD. In addition, ERT may be a therapeutic burden to patients owing to the need for regular intravenous infusions.

Oral substrate reduction therapy (SRT) with miglustat (Zavesca ${ }^{\circledR}$, Actelion Pharmaceuticals, Allschwil, Switzerland) was approved in the European Union and the USA for the treatment of adults with GD type 1 for whom ERT is unsuitable or not a therapeutic option, based on data from several clinical trials $[10,11]$. Although the clinical trial results showed beneficial effects of miglustat on key disease parameters, its problematic safety profile led to a relatively narrow label indication. Until now, there are no published data on the usage of miglustat in Korea. This case series reports our experience with miglustat in three Korean patients with GD.

\section{Materials and Methods}

Three patients (one male and two females) were given SRT at the Medical Genetics Center, Asan Medical Center Children's Hospital, Seoul, Korea, between 2010 and 2013. The patients were administered $100 \mathrm{mg}$ of miglustat three times daily. Their detailed clinical profiles, comprising age at diagnosis, presenting symptoms, accompanying signs, laboratory findings at diagnosis, GBA activity, and GBA mutations were reviewed. All patients were diagnosed with GD by a documented deficiency of GBA activity in peripheral leukocytes and mutation analysis of the $G B A$ gene using genomic DNA from peripheral blood leukocytes. Laboratory parameters including hemoglobins, platelets, chitotriosidase, acid phosphatase, and ACE were routinely evaluated for 6 to 12 months. All research protocols were approved by the Institutional Review Board of Asan Medical Center, Seoul, Korea (approval no. 2016-0797).

\section{Results}

The clinical characteristics and biomarker profiles of the patients are summarized in Table 1.

\section{Patient 1}

A 6-year-old girl was diagnosed with GD type 1 owing to progressive hepatosplenomegaly and thrombocytopenia. Diagnosis was established based on decreased GBA activity in leukocytes and bone marrow biopsy that revealed presence of Gaucher cells. Mutations in GBA (p. [lle403Thr];[Gly46Glu]) were also identified. Splenectomy was performed due to intractable thrombocytopenia. Pathology specimen of the spleen also showed Gaucher cell infiltration. ERT was initiated with imiglucerase (Cerezyme ${ }^{\circledR}$ ) for the patient at the age of 11 years. At the time of diagnosis, she had a platelet count of $234 \times 10^{3} /$ $\mathrm{mm}^{3}$, a hemoglobin concentration of $11.1 \mathrm{~g} / \mathrm{dL}_{\text {, and a liver }}$ volume of 1,137.2 $\mathrm{mL}$, as measured by computed tomography (CT). She did not exhibit any evidence of bone disease in simple radiographs and lower extremity magnetic resonance imaging (MRI). During ERT, her platelet and hemoglobin levels were stable. At the age of 25 years (after 14 years of ERT), we changed the medication to miglustat because of itching and urticaria caused by imiglucerase infusion. The patient tolerated miglustat and showed no gastrointestinal toxicities. However, miglustat treatment led to an increase in the levels of biomarkers (ACE, 29.2 to $138.9 \mathrm{U} / \mathrm{L}$; chitotriosidase, 338.5 to 2,949.7 nmol/hr/ $\mathrm{mL}$; and acid phosphatase, 11.6 to $24.8 \mathrm{U} / \mathrm{L}$ ). We checked her adherence to the medication; however, she reported that she had taken more than 70\% of the drugs prescribed. After 15 months, imiglucerase therapy was restarted due to continuous elevation of biomarker levels. After reswitching to imiglucerase, biomarker levels were normalized without any serious complications.

\section{Patient 2}

This patient was diagnosed with GD type 1 at the age of 19 years, owing to thrombocytopenia and splenomegaly. Gaucher 
Table 1. Clinical characteristics and biomarker profiles of three Gaucher disease patients treated with miglustat

\begin{tabular}{|c|c|c|c|}
\hline Characteristic & Patient 1 & Patient 2 & Patient 3 \\
\hline Age at diagnosis (yr) & 6 & 19 & 16 \\
\hline Gender & Female & Male & Female \\
\hline Type & Non-neuronopathic & Non-neuronopathic & Chronic neuronopathic \\
\hline Clinical manifestations & $\begin{array}{l}\text { Hepatomegaly, splenomegaly, } \\
\text { thrombocytopenia }\end{array}$ & $\begin{array}{l}\text { Hepatomegaly, splenomegaly, } \\
\text { thrombocytopenia, lumbar } \\
\text { spine involvement }\end{array}$ & $\begin{array}{l}\text { Seizure, intention tremor, } \\
\text { impaired cognitive function }\end{array}$ \\
\hline \multicolumn{4}{|c|}{ Hemoglobin (g/dL; normal range, 13-17 g/dL) } \\
\hline $\begin{array}{l}\text { At diagnosis } \\
\text { At the start of miglustat therapy } \\
\text { At the end of miglustat therapy }\end{array}$ & $\begin{array}{l}11.7 \\
12.5 \\
11.7\end{array}$ & $\begin{array}{l}14.2 \\
16.0 \\
16.0\end{array}$ & $\begin{array}{l}12.4 \\
11.3 \\
12.7\end{array}$ \\
\hline \multicolumn{4}{|c|}{ Platelet $\left(\times 10^{3} / \mathrm{mm}^{3}\right.$; normal range, $\left.150 \times 10^{3}-350 \times 10^{3} / \mathrm{mm}^{3}\right)$} \\
\hline $\begin{array}{l}\text { At diagnosis } \\
\text { At the start of miglustat therapy } \\
\text { At the end of miglustat therapy }\end{array}$ & $\begin{array}{l}234 \\
287 \\
173\end{array}$ & $\begin{array}{l}55 \\
80 \\
83\end{array}$ & $\begin{array}{l}114 \\
164 \\
110\end{array}$ \\
\hline Genotype & I403T/G46E & D409H/L444P & N188S/R257Q \\
\hline \multicolumn{4}{|l|}{ Biomarkers } \\
\hline \multicolumn{4}{|c|}{ Chitotriosidase (nmol/hr/mL; normal range, 4.0-76.0 nmol $/ \mathrm{hr} / \mathrm{mL}$ ) } \\
\hline $\begin{array}{l}\text { At diagnosis } \\
\text { At the start of miglustat therapy } \\
\text { At the end of miglustat therapy }\end{array}$ & $\begin{array}{c}N A \\
338.5 \\
2,949.7\end{array}$ & $\begin{array}{c}N A \\
2,497.9 \\
2,345.0\end{array}$ & $\begin{array}{l}\text { NA } \\
12.1 \\
44.6\end{array}$ \\
\hline \multicolumn{4}{|c|}{ Angiotensin-converting enzyme (U/L; normal range, 7.5-53.0 U/L) } \\
\hline $\begin{array}{l}\text { At diagnosis } \\
\text { At the start of miglustat therapy } \\
\text { At the end of miglustat therapy }\end{array}$ & $\begin{array}{r}158.0 \\
29.2 \\
138.9\end{array}$ & $\begin{array}{r}111.9 \\
121.0 \\
98.9\end{array}$ & $\begin{array}{l}89.2 \\
11.3 \\
23.3\end{array}$ \\
\hline \multicolumn{4}{|c|}{ Acid phosphatase (U/L; normal range, 6.1-11.0 U/L) } \\
\hline $\begin{array}{l}\text { At diagnosis } \\
\text { At the start of miglustat therapy } \\
\text { At the end of miglustat therapy }\end{array}$ & $\begin{array}{l}20.9 \\
11.6 \\
24.8\end{array}$ & $\begin{array}{l}17.2 \\
10.3 \\
14.4\end{array}$ & $\begin{array}{r}19.3 \\
5.1 \\
7.3\end{array}$ \\
\hline
\end{tabular}

NA, not available.

cell infiltration was noted in bone marrow biopsy. Activity of GBA in peripheral blood leukocytes was $1.07 \mathrm{nM} / \mathrm{hr} / \mathrm{mg}$ protein (normal range, 6 to $10 \mathrm{nM} / \mathrm{hr} / \mathrm{mg}$ protein), and $G B A$ gene mutations (p.[Asp409His]; [Leu444Pro]) were discovered. Lower extremity MRI showed Gaucher cell infiltration in the lumbar spine and both lower extremities. Liver and spleen volumes were 2680.7 and $1193.5 \mathrm{~mL}$, respectively, as measured by CT. ERT was initiated with imiglucerase (Cerezyme ${ }^{\circledR}$ ) in the patient at the age of 20. After two years of ERT, platelet count increased from $55 \times 10^{3}$ to $112 \times 10^{3} / \mathrm{mm}^{3}$, accompanied by decrease in the levels of acid phosphatase (17.2 to $6.7 \mathrm{U} / \mathrm{L}$ ) and ACE (111.9 to $52.6 \mathrm{U} /$ L). Furthermore, liver and spleen volumes decreased to 1,400.5 and $583 \mathrm{~mL}$, respectively (Fig. 1). Serial MRI showed slightly reduced Gaucher cell infiltration in the bone marrow of the lumbar spine and lower extremities (Fig. 1). After nine years of ERT with imiglucerase (at the age of 29 years), allergic reactions were reported. Consequently, we switched the medication to miglustat, following which, mild hand tremor and severe watery diarrhea occurred. Miglustat administration was discontinued one month later owing to persistent watery diarrhea three times a day. Reswitching to imiglucerase was uneventful without any serious complications.

\section{Patient 3}

Generalized tonic seizure first occurred in this patient at the age of 11 years, and a combination of antiepileptic drugs was used to treat epilepsy. At the age of 16 years, she was diagnosed with GD based on decreased GBA activity (11.59 pmol/min/ mg protein, normal range $20.0-80.0 \mathrm{pmol} / \mathrm{min} / \mathrm{mg}$ protein) and Gaucher cell infiltration in the bone marrow. Moreover, mutations in GBA (p.[Asn118Ser];[Arg257GIn]) were identified. At the time of diagnosis, she had a platelet count of $114 \times 10^{3} /$ $\mathrm{mm}^{3}$ and a hemoglobin concentration of $12.4 \mathrm{~g} / \mathrm{dL}$. Abdominal sonography did not show evidence of organomegaly, and brain MRI findings were within normal limits. Although the patient was on antiepileptic therapy, she was suffering from occasional events of atonic seizure and intention tremor, and her cognitive function was gradually decreasing. Imiglucerase (Cerezyme ${ }^{\circledR}$ ) 

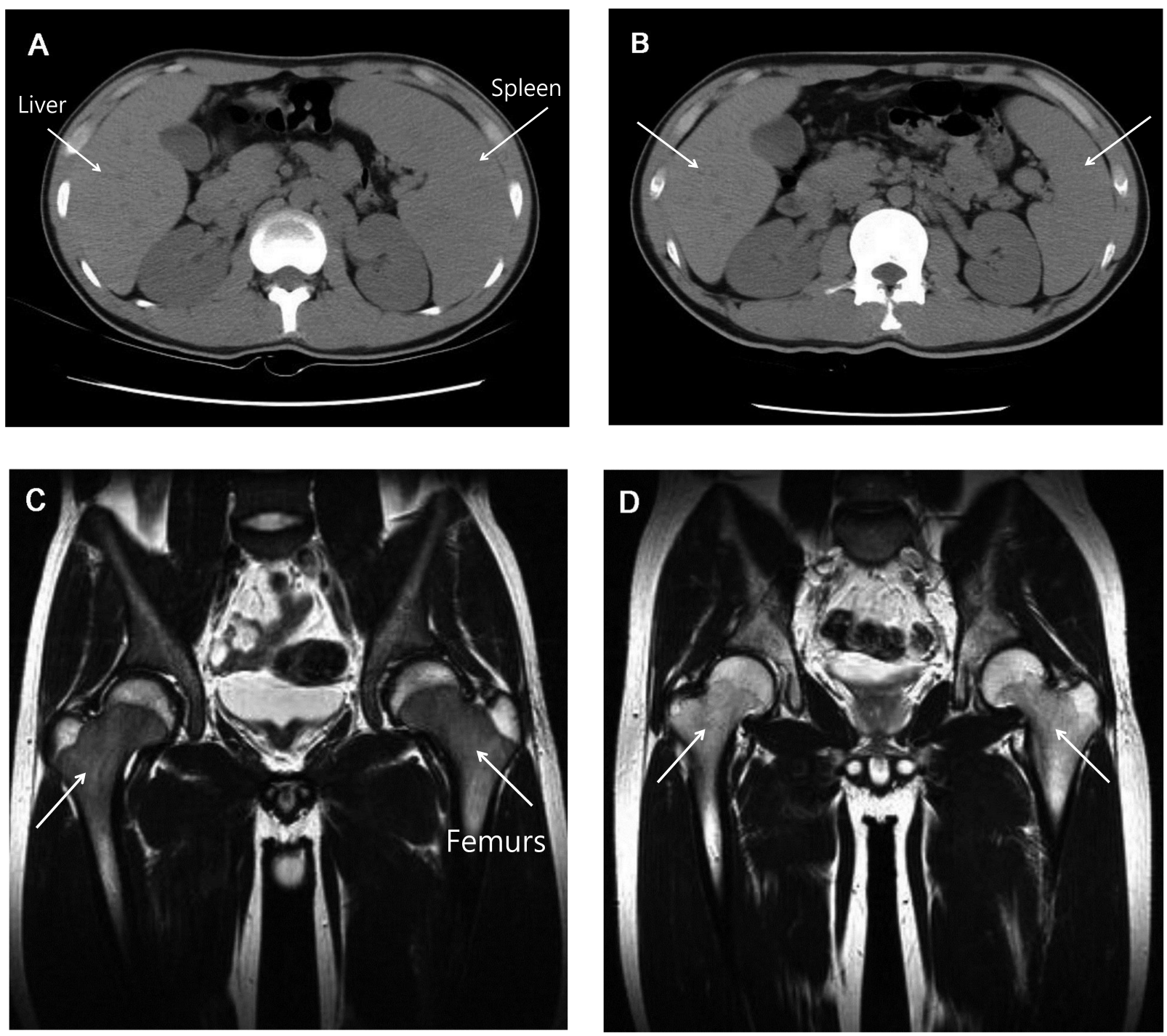

Fig. 1. Improvement of hepatosplenomegaly and skeletal symptoms after enzyme replacement therapy (ERT) in Patient 2. (A) Liver and spleen volumes measured by computed tomography at the time of diagnosis were 2,680.7 and 1,193.5 mL, respectively. (B) Liver and spleen volumes decreased to 1,400.5 and $583 \mathrm{~mL}$, respectively, after two years of ERT. (C) Lower extremity magnetic resonance imaging (MRI) at diagnosis shows decreased bone marrow signal of both femurs owing to Gaucher cell infiltration. (D) After four years of ERT, reduction of diffuse low signal intensity of both femurs was seen on T2-weighted MRI, which indicated slight reduction of Gaucher cell infiltration.

treatment was initiated immediately after diagnosis. After two years of ERT (at the age of 18 years), apparent allergic reaction was noted. Accordingly, we changed the medication to miglustat owing to itching and rash. After 17 months of miglustat administration, severe diarrhea continued for over a month that led to excessive weight loss. Eventually, we stopped the medication and restarted the imiglucerase therapy. Infectious causes were not fully excluded through laboratory tests. However, considering the long duration of diarrhea and no other associated symptoms such as abdominal pain or fever, infectious diarrhea seemed less likely. Platelet count as well as hemoglobin and other biomarker levels remained unchanged during miglustat administration. Reswitching to imiglucerase was uneventful without any serious complications.

\section{Discussion}

This case series describes our experience with GD patients who had been switched from long-term ERT to miglustat therapy. Miglustat was administered owing to allergic reactions during intravenous imiglucerase infusions. In the presented cases, reasons for discontinuation of miglustat were elevation of biomarker levels (Patient 1) and severe diarrhea, leading to weight loss (Patients 2 and 3). 
Since its introduction in 1991, ERT has been considered as the standard therapy for GD type $1[9,12]$. Over the two decades of its application, it has become evident that many of the symptoms and signs of GD such as hepatosplenomegaly, anemia, and thrombocytopenia, and often, skeletal or lung involvement, respond adequately to ERT [13]. According to previous reports, hemoglobin concentrations typically increase to normal or near normal levels within 6-12 months [9]. In thrombocytopenic patients with intact spleens, the greatest response occurred within two years, and in patients who had splenectomy, platelet counts returned to normal within 6-12 months [9]. Reduction in spleen and liver volumes usually occurs within 6 months after initiation of the therapy. Skeletal improvement may not be apparent until after two to three years of therapy. In our case, Patient 2 showed marked improvement of thrombocytopenia and hepatosplenomegaly after ERT.

Guidelines for ERT in patients with neuronopathic GD are less standardized. The recombinant enzyme cannot cross the bloodbrain barrier; and hence has limited ability to affect neurological manifestations. However, other visceral and hematologic manifestations of GD can be improved by ERT. Likewise, in Patient 3, although there were no further improvements of neurological disorders, no further aggravation of visceral and hematologic conditions was observed during the period of ERT.

Generally, ERT for GD is well tolerated. Side effects are rare and are usually related to intravenous infusion. All three cases presented with pruritis, flushing, and urticaria, and our experience with side effects was limited. These reactions were transient and re-introduction of the enzyme therapy was well tolerated without any serious adverse reaction.

Oral SRT is an alternative approach for GD treatment that reduces glycolipid accumulation by inhibiting the synthesis of the substrate of the deficient enzyme. Miglustat is a synthetic D-glucose analog that acts by inhibiting the enzyme glucosylceramide synthase. Remarkable improvements in hepatomegaly, splenomegaly, and level of biochemical markers have been observed with miglustat treatment $[10,14]$. Therefore, its ineffectiveness in Patient 1 was unexpected. Poor adherence to medication is suspected as the main reason for the drug's ineffectiveness, although this was denied by the patient.

Miglustat can cross the blood-brain barrier and is considered a standard for the management of neuronopathic forms of GD (GD types 2 and 3). However, its effects on neurological outcomes in GD type 3 have been controversial $[15,16]$. In our case, Patient 3, diagnosed with GD type 3, was on miglustat therapy for 17 months and did not show any neurologic improvements.

Side effects such as gastrointestinal complaints, tremors, and peripheral neuropathy have been reported in many cases of miglustat therapy, eventually leading to its discontinuation [17]. Similarly, Patients 2 and 3 reverted to ERT due to severe diarrhea and weight loss. According to the 9-year Intensive Safety Surveillance Scheme in miglustat (Zavesca ${ }^{\circledR}$ )-treated patients, adverse events were the most frequent cause of discontinuations, accounting for $32 \%$ of GD type 1 patients and $11 \%$ of patients with other diseases. Other reasons for discontinuation were disease progression and perceived lack of efficacy [14]. Moreover, considering the essential roles of lipids in cells, SRT may affect many cellular functions due to long term reduction of glycosphingolipid levels [18].

Eliglustat tartrate (GENZ-112638) is a new glucosylceramide inhibitor with stronger inhibitory potency than miglustat. Eliglustat does not cross the blood-brain barrier. In contrast to miglustat, eliglustat does not potently inhibit intestinal glycosidases. Hence, the gastrointestinal side effects observed with miglustat are largely avoided [19]. Trial results of eliglustat suggested its efficacy and safety, both in naïve GD type 1 patients and in patients who switched over from ERT [20,21]. Eliglustat showed positive characteristics as an oral treatment for GD type 1, with satisfactory clinical effects on visceral and hematological symptoms. Although the side effects reported were generally mild, some patients showed electrocardiogram abnormalities [22].

Despite the small number of patients, this is the first to report on SRT in Korea. In the Korean population, neuronopathic GD is more common than non-neuronopathic GD in comparison with Caucasian populations. According to a previous report, $39 \%$ (14/36) of Korean GD patients were classified as the neuronopathic type [23]. Our experience with adult GD type 1 patients indicates that serious complications including severe diarrhea and peripheral neuropathy, led to withdrawal of the medication. Thus, careful consideration should be taken before prescribing miglustat. In addition, despite its ability to cross the blood-brain barrier, we could not suggest the use of miglustat against neuronopathic GD (types 2 and 3) based on our experience and review of the previous clinical trials. Eliglustat, the new agent with lower incidence of adverse reactions, may be considered as an alternative against non-neuronopathic forms of GD. However, its suitability needs to be validated in a larger cohort of patients with long-term observation. 


\section{Acknowledgements}

This study was supported by a grant from the National Research Foundation of Korea, which was funded by the Ministry of Education, Science, and Technology (NRF-2016M3A9B4915706).

\section{References}

1. Sidransky E. Gaucher disease: complexity in a "simple" disorder. Mol Genet Metab 2004;83:6-15.

2. Zimran A. How I treat Gaucher disease. Blood 2011;118:1463-71.

3. Cabrera-Salazar MA, O'Rourke E, Henderson N, Wessel H, Barranger JA. Correlation of surrogate markers of Gaucher disease. Implications for long-term follow up of enzyme replacement therapy. Clin Chim Acta 2004;344:101-7.

4. Lee JY, Lee $B H$, Kim GH, Jung CW, Lee J, Choi JH, et al. Clinical and genetic characteristics of Gaucher disease according to phenotypic subgroups. Korean J Pediatr 2012;55:48-53.

5. Zimran A, Elstein D, Levy-Lahad E, Zevin S, Hadas-Halpern I, Bar-Ziv $Y$, et al. Replacement therapy with imiglucerase for type 1 Gaucher's disease. Lancet 1995;345:1479-80.

6. Choi JH, Lee BH, Ko JM, Sohn YB, Lee JS, Kim GH, et al. A phase 2 multi-center, open-label, switch-over trial to evaluate the safety and efficacy of Abcertin ${ }^{\circledR}$ in patients with type 1 Gaucher disease. J Korean Med Sci 2015;30:378-84.

7. Zimran A, Altarescu G, Philips M, Attias D, Jmoudiak M, Deeb $M$, et al. Phase $1 / 2$ and extension study of velaglucerase alfa replacement therapy in adults with type 1 Gaucher disease: 48-month experience. Blood 2010;115:4651-6.

8. Zimran A, Brill-Almon $E_{1}$ Chertkoff $R_{1}$ Petakov $M$, Blanco-Favela $F_{1}$ Muñoz ET, et al. Pivotal trial with plant cell-expressed recombinant glucocerebrosidase, taliglucerase alfa, a novel enzyme replacement therapy for Gaucher disease. Blood 2011;118:5767-73.

9. Weinreb NJ, Charrow J, Andersson HC, Kaplan P, Kolodny EH, Mistry P, et al. Effectiveness of enzyme replacement therapy in 1028 patients with type 1 Gaucher disease after 2 to 5 years of treatment: a report from the Gaucher Registry. Am J Med 2002;113:112-9.

10. Cox T, Lachmann R, Hollak C, Aerts J, van Weely S, Hrebicek $M$, et al. Novel oral treatment of Gaucher's disease with $\mathrm{N}$-butyldeoxynojirimycin (OGT 918) to decrease substrate biosynthesis. Lancet 2000;355:1481-5.

11. Heitner R, Elstein D, Aerts J, Weely Sv, Zimran A. Low-dose N-butyldeoxynojirimycin (OGT 918) for type I Gaucher disease. Blood
Cells Mol Dis 2002;28:127-33.

12. Weinreb NJ, Goldblatt J, Villalobos J, Charrow J, Cole JA, Kerstenetzky $M$, et al. Long-term clinical outcomes in type 1 Gaucher disease following 10 years of imiglucerase treatment. J Inherit Metab Dis 2013;36:543-53.

13. Elstein $D$, Zimran A. Review of the safety and efficacy of imiglucerase treatment of Gaucher disease. Biologics 2009;3:407-17.

14. Brand M, Muller A, Alsop J, van Schaik IN, Bembi B, Hughes D. Results from a 9-year Intensive Safety Surveillance Scheme (IS(3) ) in miglustat (Zavesca( $\left.{ }^{\circledR}\right)$ )-treated patients. Pharmacoepidemiol Drug Saf 2015;24:329-33.

15. Cox-Brinkman J, van Breemen MJ, van Maldegem BT, Bour L, Donker WE, Hollak $C E$, et al. Potential efficacy of enzyme replacement and substrate reduction therapy in three siblings with Gaucher disease type III. J Inherit Metab Dis 2008;31:745-52.

16. Schiffmann R, Fitzgibbon EJ, Harris $C$, DeVile $C$, Davies EH, Abel L, et al. Randomized, controlled trial of miglustat in Gaucher's disease type 3. Ann Neurol 2008;64:514-22.

17. Kuter DJ, Mehta A, Hollak CE, Giraldo P, Hughes D, Belmatoug N, et al. Miglustat therapy in type 1 Gaucher disease: clinical and safety outcomes in a multicenter retrospective cohort study. Blood Cells Mol Dis 2013;51:116-24

18. Futerman $A H$, Hannun $Y A$. The complex life of simple sphingolipids. EMBO Rep 2004:5:777-82.

19. McEachern KA, Fung J, Komarnitsky S, Siegel CS, Chuang WL, Hutto $E_{1}$ et al. A specific and potent inhibitor of glucosylceramide synthase for substrate inhibition therapy of Gaucher disease. Mol Genet Metab 2007:91:259-67.

20. Lukina E, Watman N, Dragosky M, Pastores GM, Arreguin EA Rosenbaum $H_{1}$ et al. Eliglustat, an investigational oral therapy for Gaucher disease type 1: Phase 2 trial results after 4 years of treatment. Blood Cells Mol Dis 2014;53:274-6.

21. Mistry PK, Lukina $E$, Ben Turkia $H$, Amato D, Baris $H$, Dasouki $M$ et al. Effect of oral eliglustat on splenomegaly in patients with Gaucher disease type 1: the ENGAGE randomized clinical trial. JAMA 2015:313:695-706

22. Peterschmitt MJ, Burke A, Blankstein L, Smith SE, Puga AC, Kramer $W G$, et al. Safety, tolerability, and pharmacokinetics of eliglustat tartrate (Genz-112638) after single doses, multiple doses, and food in healthy volunteers. J Clin Pharmacol 2011;51:695-705

23. Jeong SY, Park SJ, Kim HJ. Clinical and genetic characteristics of Korean patients with Gaucher disease. Blood Cells Mol Dis 2011;46:11-4. 